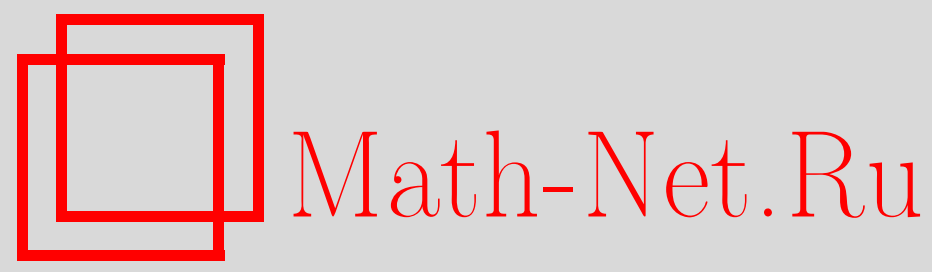

Е. А. Снегова, Критерий сводимости задачи об опасной близости к одномерному интервальному поиску, Дискрет. матем., 2011, том 23, выпуск 3, 138-159

DOI: https://doi.org/10.4213/dm1157

Использование Общероссийского математического портала Math-Net.Ru подразумевает, что вы прочитали и согласны с пользовательским соглашением http://www . mathnet.ru/rus/agreement

Параметры загрузки:

IP : 54.157 .27 .8

26 апреля 2023 г., 12:53:52 


\title{
Критерий сводимости задачи об опасной близости к одномерному интервальному поиску
}

\author{
(C) 2011 г. $\quad$ Е. А. Снегова
}

\begin{abstract}
В работе рассматривается задача о поиске движущихся объектов, которые могут столкнуться с движущимся объектом-запросом, где под столкновением понимается нахождение объектов в опасной близости. Приведен критерий сводимости данной задачи к задаче одномерного интервального поиска.
\end{abstract}

\section{1. Введение}

Традиционно в системах управления базами данных предполагается, что каждый движущийся объект имеет свою собственную независимую скорость и собственное направление движения, то есть объекты движутся хаотично. В данной статье рассматриваются потоки движущихся объектов и конфликты между потоками.

Приведем пример приложения задачи об опасной близости.

Рассмотрим квадрат над аэропортом и два типа самолетов: самолеты одного типа (запросы) движутся с юга на север внутри квадрата, а самолеты другого типа (объекты) движутся с запада на восток также внутри квадрата. Каждый самолет, когда он появляется на границе квадрата, объявляет координаты появления и время своего появления. Задача об опасной близости заключается в перечислении для каждого нового самолетазапроса тех и только тех самолетов-объектов, которые будут в некоторый момент времени в процессе своего движения на расстоянии не более, чем $\rho$ от запроса по Манхэттену.

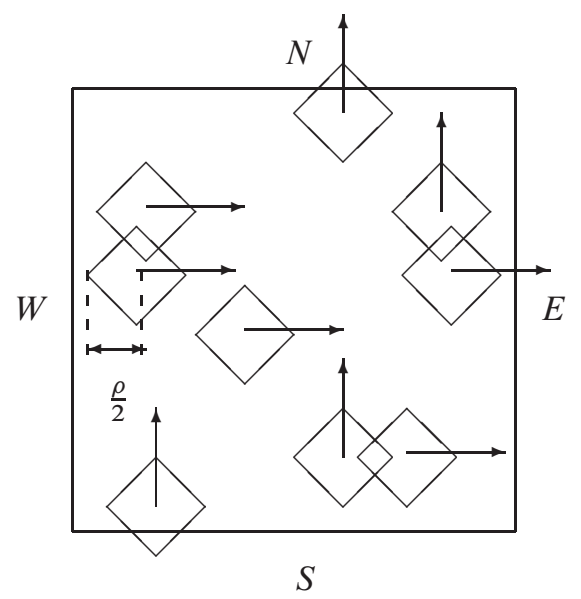

Рис. 1. Движение объектов и запросов в задаче об опасной близости 
Расстояние $\rho$ может быть интерпретировано следующим образом: рассмотрим самолет, представляющий собой круг радиуса $\rho$ в Манхэттеновской метрике, как это показано на рис. 1. Задача об опасной близости заключается в том, чтобы для каждого самолетазапроса перечислить те и только те самолеты-объекты, которые будут пересекаться с запросом в процессе своего движения.

В основной части этой статьи приводится формальная постановка задачи об опасной близости, формулируется и доказывается критерий сводимости данной задачи к задаче одномерного интервального поиска. Для случая задачи об опасной близости, сводящейся к задаче одномерного интервального поиска, получены логарифмические оценки сложности поиска, вставки и удаления, и линейный объем памяти.

Автор выражает глубокую благодарность Э. Э. Гасанову за постановку задачи и помощь в работе.

\section{2. Обзор литературы}

Задача об опасной близости тесно связана с задачей поиска объекта, ближайшего к запросу. Рассмотрим существующие методы решения последней задачи и сравним их с решением задачи об опасной близости в том случае, когда она сводится к задаче одномерного интервального поиска.

В большей части методов решения задачи поиска объекта, ближайшего к запросу, используются структуры данных, основанные на конструкции $R$-дерева [1].

Первая группа методов относится к случаю статической базы данных, состоящей из неподвижных точек, и статического точечного запроса [2]. Идея этих методов заключается в многоступенчатой проверке данных, происходящей до тех пор, пока не достигается подходящий радиус. Например, в статье [2] разработан алгоритм, состоящий из нескольких предварительных и одного финального этапа отбора.

Для движущихся объектов и статических запросов в [3] разработан алгоритм, позволяющий получать ответ на запрос о положении объектов в пространстве. Структура данных для этого алгоритма основана на $R^{*}$-дереве [4] - модификации $R$-дерева. В [5] упрощен упомянутый алгоритм без потери в эффективности, а в [6] приведено его расширение на случай непрерывно движущихся объектов.

В [7] предложено решение задачи нахождения $k$ ближайших к запросу объектов. Большинство алгоритмов для случая непрерывного движения объектов основываются на повторных применениях алгоритмов для решения обычной задачи о нахождении ближайшего соседа, что приносит значительные издержки (см., например, [8]). В отличие от этого подхода, в [9] разработаны методы, которые решают данную задачу путем формирования единого запроса для всей базы данных.

Случай движущихся объектов и движущихся запросов - самый близкий к задаче об опасной близости. В [10] введено понятие структуры, позволяющей в режиме реального времени определять текущее положение объектов.

Все методы, приведенные выше, основаны на структуре $R$-дерева или ее модификации, предназначенной для хранения двух- или трехмерных объектов. Данные методы полезны в случае хаотического движения объектов. Во всех работах нет аналитических оценок сложности, и авторы показывают эффективность их результатов с помощью экспериментов. Однако нетрудно заметить, что в худшем случае оценки не более чем линейные от размера базы данных.

Случай предопределенного движения объектов рассмотрен в [11]. Автор предложил алгоритмы для поиска самых близких к запросу и самых быстрых относительно запроса 
объектов и также алгоритмы для вычисления выпуклой оболочки движущихся точек. Для некоторых алгоритмов получены линейные нижние оценки сложностей.

В данной работе также рассматривается случай предопределенного движения объектов. Данные о законах движения объектов и запросов позволяют нам в каждом конкретном случае определить, может ли задача об опасной близости быть сведена к задаче одномерного интервального поиска.

В ряде работ было разработано множество методов решения задачи одномерного интервального поиска, например, [12-20]. Для динамического случая наиболее подходящим является метод, основанный на структуре 2-3 дерева [21]. Данный метод описан в [22, 23]. Соответствующий алгоритм имеет логарифмическую сложность поиска, вставки и удаления, что значительно меньше, чем оценки, полученные в [11].

\section{3. Постановка задачи}

Пусть заданы две функции

$$
\begin{array}{ll}
f: & {\left[0, \tau_{\max }\right] \rightarrow[-\rho, 1+\rho],} \\
f_{q}: & {\left[0, \tau_{\max }^{q}\right] \rightarrow[-\rho, 1+\rho],}
\end{array}
$$

называемые законами движения объектов и объектов-запросов, соответственно. Функции являются непрерывными и строго монотонными на всей области определения и удовлетворяют условиям

$$
f(0)=f_{q}(0)=-\rho, \quad f\left(\tau_{\max }\right)=f_{q}\left(\tau_{\max }^{q}\right)=1+\rho .
$$

Считаем, что на плоскости имеется счетное множество объектов, движущихся таким образом, что их координаты в зависимости от времени задаются парой $\left(f\left(t-t_{i}\right), y_{i}\right)$, где $i \in \mathbf{N}, y_{i} \in[0,1]$, а $t_{i}$ образуют строго возрастающую последовательность вещественных чисел. Аналогично, движение объекта-запроса задается парой $\left(x, f_{q}\left(t-t_{q}\right)\right)$, где $x \in[0,1]$, $t_{q}$ - вещественное число.

Библиотекой $V\left(t_{q}\right)$ назовем множество объектов, находящихся в текущий момент времени $t_{q}$ внутри прямоугольника $[-\rho, 1+\rho] \times[0,1]$, то есть

$$
V_{\left(t_{q}\right)}=\left\{\left(t_{i}, y_{i}\right): t_{q} \in\left[t_{i}, t_{i}+\tau_{\max }\right], y_{i} \in[0,1]\right\} .
$$

Вместо $V\left(t_{q}\right)$ будем писать просто $V$, понимая под этим множество всех объектов, находящихся в текущий момент времени внутри квадрата. Через $|V|$ обозначим число объектов в этой библиотеке.

В задаче требуется для произвольного запроса перечислить все объекты из библиотеки, с которыми он в процессе своего движения будет находиться на расстоянии меньшем, чем $\rho$ по Манхэттену, то есть ответом на запрос $q=\left(t_{q}, x\right)$ при библиотеке $V=V\left(t_{q}\right)$ и расстоянии опасной близости $\rho$ является множество

$$
J(\rho, q, V)=\left\{o_{i} \in V|\exists t:| f\left(t-t_{i}\right)-x|+| y_{i}-f_{q}\left(t-t_{q}\right) \mid \leqslant \rho\right\} .
$$

Тройку $\left(f, f_{q}, \rho\right)$ будем называть задачей об опасной близости.

Задачей одномерного интервального поиска назовем пару $(I, Z)$, где библиотека $Z-$ конечное подмножество множества действительных чисел $\mathbf{R}$, а множество запросов $I-$ есть множество всех интервалов с концами из R. Содержательно эта задача состоит в 
том, чтобы для произвольного запроса $p \in I$ перечислить все те и только те точки из $Z$, которые попадают в интервал $p$.

Ответ на запрос $p \in I$ при библиотеке $Z$ в задаче одномерного интервального поиска есть множество

$$
J(p, Z)=\{z \in Z: z \in p\} .
$$

Будем говорить, что задача об опасной близости $\left(f, f_{q}, \rho\right)$ сводится с задаче одномерного интервального поиска, если существуют такие отображения $\varphi, \varphi_{1}, \varphi_{2}: \mathbf{R} \times[0,1] \rightarrow \mathbf{R}$, что для любой библиотеки $V=V\left(t_{q}\right)$, любого запроса $q=\left(t_{q}, x\right)$ и любого объекта $o \in V$

$$
o \in J(\rho, q, V) \Longleftrightarrow \varphi(o) \in J\left(\left[\varphi_{1}(q), \varphi_{2}(q)\right], Z\right),
$$

где

$$
Z=\{\varphi(o): o \in V\} .
$$

Тройку $\left(\varphi, \varphi_{1}, \varphi_{2}\right)$ будем называть функциями сведения.

Введем обозначения

$$
\begin{aligned}
F_{L}(x, y) & =\min _{\xi \in[0, \rho]}\left(f_{q}^{-1}(y+\xi-\rho)-f^{-1}(x+\xi)\right), \\
F_{R}(x, y) & =\max _{\xi \in[-\rho, 0]}\left(f_{q}^{-1}(y+\xi+\rho)-f^{-1}(x+\xi)\right), \\
X_{R} & =\left\{x \in[0,1]: \exists y F_{R}(x, y) \leqslant 0\right\}, \\
X_{L} & =\left\{x \in[0,1]: \exists y F_{L}(x, y) \leqslant 0\right\}, \\
Y_{L} & =\left\{y \in[0,1]: \exists x F_{L}(x, y) \leqslant 0\right\} .
\end{aligned}
$$

Теорема 1. Задача об опасной близости $\left(f, f_{q}, \rho\right)$ сводится к задаче одномерного интервального поиска тогда и только тогда, когда существуют функции $\psi, \psi_{L}, \psi_{R}:[0,1] \rightarrow \mathbf{R}$, такие, что для любой пары $(x, y)$, для которой выполнено условие $F_{L}(x, y) \leqslant 0$, справедливо равенство

$$
F_{L}(x, y)=\psi(y)+\psi_{L}(x),
$$

а для любой пары $(x, y)$, такой, что $F_{R}(x, y) \leqslant 0$, справедливо равенство

$$
F_{R}(x, y)=\psi(y)+\psi_{R}(x) .
$$

При этом, если выполнень условия (6) и (7) $и$

$$
\begin{aligned}
& \psi^{*}(y)= \begin{cases}\psi(y), & \text { если } y \in Y_{L}, \\
F_{L}(0,1), & \text { если } y \notin Y_{L},\end{cases} \\
& \psi_{R}^{*}(x)= \begin{cases}\psi_{R}(x), & \text { если } x \in X_{R}, \\
F_{R}(0,1), & \text { если } x \notin X_{R},\end{cases} \\
& \psi_{L}^{*}(x)= \begin{cases}\psi_{L}(x), & \text { если } x \in X_{L}, \\
F_{L}(0,1), & \text { если } x \notin X_{L},\end{cases}
\end{aligned}
$$

то функичи сведения $\left(\varphi, \varphi_{1}, \varphi_{2}\right)$ имеют вид

$$
\begin{aligned}
\varphi\left(t_{i}, y_{i}\right) & =t_{i}-\psi^{*}\left(y_{i}\right), \\
\varphi_{1}\left(t_{q}, x\right) & =t_{q}+\psi_{L}^{*}(x), \\
\varphi_{2}\left(t_{q}, x\right) & =t_{q}+\psi_{R}^{*}(x) .
\end{aligned}
$$




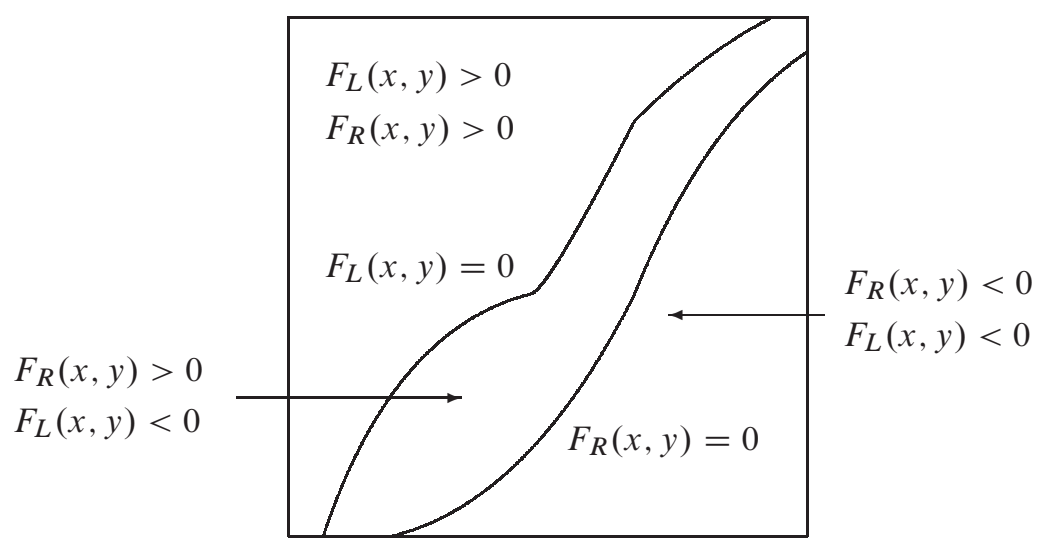

Рис. 2. Области знакопостоянства функций $F_{L}(x, y), F_{R}(x, y)$

Области $F_{L}(x, y) \leqslant 0, F_{R}(x, y) \leqslant 0$ изображены на рис. 2 .

Теорема 1 в случае, когда выполнены условия (6) и (7), позволяет решать задачу об опасной близости путем сведения ее к динамической задаче одномерного интервального поиска следующим образом.

Пусть дана динамическая база данных вещественных точек для решения задачи одномерного интервального поиска. Если в текущий момент $t$ на границе области наблюдения появляется объект $o_{i}=\left(t_{i}, y_{i}\right)$, то есть $t=t_{i}$, то точка $\varphi\left(t_{i}, y_{i}\right)$ заносится в базу данных. Если в текущий момент $t$ объект $o_{i}=\left(t_{i}, y_{i}\right)$ выходит из области наблюдения, то есть $t=t_{i}+\tau_{\max }$, то точка $\varphi\left(t_{i}, y_{i}\right)$ удаляется из базы данных. Если в текущий момент $t$ появляется запрос $q=\left(t_{q}, x\right)$, то есть $t=t_{q}$, то в базе данных ищутся все точки, попадающие в интервал $\left[\varphi_{1}\left(t_{q}, x\right), \varphi_{2}\left(t_{q}, x\right)\right]$.

В работах $[22,23]$ подробно описывается способ решения задачи об опасной близости путем сведения ее к динамической задаче одномерного интервального поиска. Там же показано, что динамическая задача одномерного интервального поиска имеет логарифмическую относительного общего числа объектов в базе данных сложность поиска (без учета сложности перечисления ответа), вставки и удаления, и линейный объем памяти. Так в [22] рассматривался случай фиксированных скоростей объектов, то есть $f(t)=v t$, a $f_{q}(t)=v_{q} t$, а в [23] рассматривался случай, когда $f^{\prime}\left(t+t^{\prime}\right)-f_{q}^{\prime}(t) \leqslant 0$ для любого $t \in\left[0, \tau_{\max }^{q}\right]$ и любого $t^{\prime}$, такого, что $t+t^{\prime} \in\left[0, \tau_{\max }\right]$. Можно проверить, что оба этих случая подпадают под условия теоремы 1.

Если воспользоваться структурами данных и алгоритмами, описанными в [21], то помимо логарифмического времени поиска, вставки и удаления в худшем случае, можно добиться константного времени выполнения этих операций в среднем.

\section{4. Доказательство основного результата}

\section{1. Свойства функций $F_{R}(x, y), F_{L}(x, y)$}

Перечислим некоторые свойства функций $F_{L}(x, y), F_{R}(x, y)$.

Свойство 1. Функции $F_{R}(x, y)$ и $F_{L}(x, y)$ строго возрастают по $y$ и убывают по $x$. 
Доказательство. Покажем, для примера, что $F_{L}(x, y)$ возрастает по $y$. Возьмем $y_{1}<y_{2}$ и фиксируем $\xi_{2}$ такой, что $\xi_{2} \in \arg \min _{\xi \in[0, \rho]}\left[f_{q}^{-1}(y+\xi-\rho)-f^{-1}(x+\xi)\right]$. Тогда верна цепочка неравенств

$$
\begin{aligned}
F_{L}\left(x, y_{2}\right) & =f_{q}^{-1}\left(y_{2}+\xi_{2}-\rho\right)-f^{-1}\left(x+\xi_{2}\right) \\
& >f_{q}^{-1}\left(y_{1}+\xi_{2}-\rho\right)-f^{-1}\left(x+\xi_{2}\right) \\
& \geqslant \min _{\xi \in[0, \rho]}\left[f_{q}^{-1}\left(y_{1}+\xi-\rho\right)-f^{-1}(x+\xi)\right] .
\end{aligned}
$$

Отсюда вытекает, что $F_{L}(x, y)$ возрастает по $y$.

Остальные случаи доказываются аналогично. Свойство 1 доказано.

Свойство 2. Функции $F_{R}(x, y), F_{L}(x, y)$ непрерывны по $y$ при фиксированном $x$ и непрерывны по $x$ при фиксированном $y$.

Доказательство. Рассмотрим последовательность $x_{n}$, стремящуюся к $x$. Тогда

$$
\begin{aligned}
\lim _{x_{n} \rightarrow x} F_{L}\left(x_{n}, y\right) & =\lim _{x_{n} \rightarrow x} \min _{\xi \in[0, \rho]}\left(f_{q}^{-1}(y+\xi-\rho)-f^{-1}\left(x_{n}+\xi\right)\right) \\
& =\min _{\xi \in[0, \rho]}\left(f_{q}^{-1}(y+\xi-\rho)-f^{-1}(x+\xi)\right)
\end{aligned}
$$

в силу непрерывности функции $f^{-1}$.

Остальные случаи доказываются аналогично. Свойство 2 доказано.

Свойство 3. Для любой пары $(x, y) \in[0,1]^{2}$

$$
F_{L}(x, y)+\tau_{\max }>0 .
$$

Доказательство. Справедливы оценки

$$
\begin{aligned}
F_{L}(x, y) \geqslant F_{L}(1,0) & =\min _{\xi \in[0, \rho]}\left(f_{q}^{-1}(\xi-\rho)-f^{-1}(1+\xi)\right) \\
& \geqslant f_{q}^{-1}(0)-f^{-1}(1+\rho)>-f^{-1}(1+\rho)=\tau_{\max }
\end{aligned}
$$

так как $f_{q}^{-1}(0)>f_{q}^{-1}(-\rho)=0$.

Свойство 3 доказано.

Свойство 4. Для любой пары $(x, y) \in[0,1]^{2}$

$$
F_{R}(x, y)-F_{L}(x, y)>0 .
$$

Доказательство. Заметим, что

$$
\begin{aligned}
& F_{R}(x, y) \geqslant f_{q}^{-1}(y+\rho)-f^{-1}(x), \\
& F_{L}(x, y) \leqslant f_{q}^{-1}(y-\rho)-f^{-1}(x),
\end{aligned}
$$

то есть

$$
F_{R}(x, y)-F_{L}(x, y) \geqslant f_{q}^{-1}(y+\rho)-f_{q}^{-1}(y-\rho)>0,
$$

так как функция $f_{q}^{-1}$ строго возрастает. Свойство 4 доказано. 


\section{2. Основная лемма}

Лемма 1. Объект $o_{i}=\left(t_{i}, y_{i}\right)$ из библиотеки $V=V\left(t_{q}\right)$ принадлежит ответу на запрос $q=\left(t_{q}, x\right)$, то есть $\left.o_{i} \in J(\rho, q, V)\right)$ тогда и только тогда, когда

$$
t_{i}-t_{q} \in\left[F_{L}\left(x, y_{i}\right), F_{R}\left(x, y_{i}\right)\right] .
$$

Доказательство. Пусть $o_{i} \in V$. Тогда по определению

$$
o_{i} \in J(\rho, q, V) \Longleftrightarrow \exists t:\left|f\left(t-t_{i}\right)-x\right|+\left|f_{q}\left(t-t_{q}\right)-y_{i}\right| \leqslant \rho .
$$

Введем обозначение

$$
\xi=f\left(t-t_{i}\right)-x
$$

Тогда

$$
o_{i} \in J(\rho, q, V) \Longleftrightarrow \exists \xi: g(\xi)=|\xi|+\left|f_{q}\left(f^{-1}(x+\xi)+t_{i}-t_{q}\right)-y_{i}\right| \leqslant \rho .
$$

Рассмотрим два случая.

Случай 1: $f\left(f_{q}^{-1}\left(y_{i}\right)+t_{q}-t_{i}\right)-x \geqslant 0$. Этот случай соответствует ситуации, когда объект пролетел точку $x$ к моменту, когда запрос достиг точки $y_{i}$. Раскрывая это неравенство, получаем, что

$$
\begin{aligned}
f_{q}^{-1}\left(y_{i}\right)+t_{q}-t_{i} & \geqslant f^{-1}(x), \\
t_{i}-t_{q} \leqslant f_{q}^{-1}\left(y_{i}\right)-f^{-1}(x) & \leqslant f_{q}^{-1}\left(y_{i}+\rho\right)-f^{-1}(x) \leqslant F_{R}\left(x, y_{i}\right) .
\end{aligned}
$$

Нетрудно заметить, что в данном случае минимум $g(\xi)$ нужно искать на отрезке $\left[0, f\left(f_{q}^{-1}\left(y_{i}\right)+t_{q}-t_{i}\right)-x\right]$, на котором

$$
g(\xi)=\xi-f_{q}\left(f^{-1}(x+\xi)+t_{i}-t_{q}\right)+y_{i} \leqslant \rho .
$$

Последнее равносильно неравенству

$$
t_{i}-t_{q} \geqslant f_{q}^{-1}\left(y_{i}+\xi-\rho\right)-f^{-1}(x+\xi) .
$$

Теперь покажем, что условие $\xi \in\left[0, f\left(f_{q}^{-1}\left(y_{i}\right)+t_{q}-t_{i}\right)-x\right]$ эквивалентно условию $\xi \in$ $[0, \rho]$. Если $f\left(f_{q}^{-1}\left(y_{i}\right)+t_{q}-t_{i}\right)-x \leqslant \rho$, то возьмем $\xi_{1}=f\left(f_{q}^{-1}\left(y_{i}\right)+t_{q}-t_{i}\right)-x$ и, очевидно, $g\left(\xi_{1}\right) \leqslant \rho$, а если $f\left(f_{q}^{-1}\left(y_{i}\right)+t_{q}-t_{i}\right)-x>\rho$, то для любого $\xi_{2} \in\left(\rho, f\left(f_{q}^{-1}\left(y_{i}\right)+t_{q}-t_{i}\right)-x\right]$ справедливо неравенство $g\left(\xi_{2}\right) \geqslant \xi_{2}>\rho$, то есть в этом случае искомая точка минимума лежит на отрезке $[0, \rho]$.

Случай 2: $f\left(f_{q}^{-1}\left(y_{i}\right)+t_{q}-t_{i}\right)-x<0$. Этот случай соответствует ситуации, когда запрос достиг точки $y_{i}$, а объект еще не долетел до точки $x$. Раскрывая это неравенство, получаем, что

$$
\begin{gathered}
f_{q}^{-1}\left(y_{i}\right)+t_{q}-t_{i}<f^{-1}(x), \\
t_{i}-t_{q}>f_{q}^{-1}\left(y_{i}\right)-f^{-1}(x) \geqslant f_{q}^{-1}\left(y_{i}-\rho\right)-f^{-1}(x) \geqslant F_{L}\left(x, y_{i}\right) .
\end{gathered}
$$


Нетрудно заметить, что в данном случае минимум $g(\xi)$ нужно искать на отрезке $\left[f\left(f_{q}^{-1}\left(y_{i}\right)+t_{q}-t_{i}\right)-x, 0\right]$, на котором

$$
g(\xi)=-\xi-y_{i}+f_{q}\left(f^{-1}(x+\xi)+t_{i}-t_{q}\right) \leqslant \rho .
$$

Последнее равносильно неравенству

$$
t_{i}-t_{q} \leqslant f_{q}^{-1}\left(y_{i}+\xi+\rho\right)-f^{-1}(x+\xi) .
$$

Аналогично первому случаю, условие $\xi \in\left[f\left(f_{q}^{-1}\left(y_{i}\right)+t_{q}-t_{i}\right)-x, 0\right]$ можно заменить на условие $\xi \in[-\rho, 0]$.

Лемма 1 доказана.

\section{3. Достаточность}

Пусть выполнены условия теоремы 1 и существуют некоторые функции $\psi, \psi_{L}, \psi_{R}$ : $[0,1] \rightarrow \mathbf{R}$, такие, что выполнены условия (6), (7). Пусть $X_{R}, X_{L}$, и $Y_{L}-$ множества, задаваемые соотношениями (3), (4) и (5) соответственно.

Заметим, что $X_{R} \subseteq X_{L}$ и некоторые из этих множеств могут быть пустыми. Поскольку функции $F_{R}(x, y), F_{L}(x, y)$ возрастают и непрерывны по $y$ при фиксированном $x$, но убывают и непрерывны по $x$ при фиксированном $y$, то для множества $X_{R}$ верно, что если оно не пусто, то $X_{R}=\left[\alpha_{x R}, 1\right]$ для некоторого положительного $\alpha_{x R}$.

Аналогично, если $X_{L} \neq \varnothing$, то $X_{L}=\left[\alpha_{x L}, 1\right]$, и если $Y_{L} \neq \varnothing$, то $Y_{L}=\left[0, \alpha_{y L}\right)$.

Пусть $\psi^{*}, \psi_{R}^{*}, \psi_{L}^{*},:[0,1] \rightarrow \mathbf{R}-$ функции, определяемые соотношениями (8), (9) и (10) соответственно.

По определению и лемме $1, \psi^{*}$ не убывает на всей области определения, а $\psi_{L}^{*}, \psi_{R}^{*}$ не возрастают, и при этом $\psi_{L}^{*}(x) \leqslant \psi_{R}^{*}(x)$ для любого $x$ из отрезка $[0,1]$.

Покажем, что если выполнены условия теоремы 1 , то для любой пары $(x, y)$, такой, что $F_{L}(x, y)>0$, справедливо неравенство

$$
\psi^{*}(y)+\psi_{L}^{*}(x)>0,
$$

а для любой пары $(x, y)$, такой, что $F_{R}(x, y)>0$, справедливо неравенство

$$
\psi^{*}(y)+\psi_{R}^{*}(x)>0 .
$$

Докажем (15). Пусть $F_{L}(x, y)>0$. Возможны два случая.

1. $y \leqslant \alpha_{y L}$. Следовательно, существует $x_{1}>x$ такой, что $F_{L}\left(x_{1}, y\right) \leqslant 0$. Из непрерывности и убывания $F_{L}(x, y)$ по $x$ при фиксированном $y$ следует, что существует $x_{0} \in\left[x, x_{1}\right]$, такой, что

$$
0=F_{L}\left(x_{0}, y\right)=\psi_{L}^{*}\left(x_{0}\right)+\psi^{*}(y)<\psi_{L}^{*}(x)+\psi^{*}(y) .
$$

Последнее неравенство выполняется в силу убывания функции $\psi_{L}(x)$.

2. $y>\alpha_{y L}$. Следовательно,

$$
\psi_{L}^{*}(x)+\psi^{*}(y)=\psi_{L}^{*}(x)+F_{L}(0,1) \geqslant \psi_{L}^{*}(x)+\psi^{*}\left(\alpha_{y L}\right)>0 .
$$

Последнее неравенство выполняется в силу предыдущего пункта. 
Аналогично доказывается неравенство (16).

Пусть $\varphi, \varphi_{1}, \varphi_{2}:[0,1] \rightarrow \mathbf{R}$ - отображения, задаваемые соотношениями (11), (12) и (13) соответственно. Покажем, что для произвольного запроса $q=\left(t_{q}, x\right)$ и произвольного объекта $o_{i}=\left(t_{i}, y_{i}\right)$ из библиотеки $V=V\left(t_{q}\right)$ выполнено соотношение

$$
o_{i} \in J(\rho, q, V) \Longleftrightarrow \varphi\left(o_{i}\right) \in J\left(\left[\varphi_{1}(q), \varphi_{2}(q)\right], Z\right),
$$

где

$$
Z=\{\varphi(o): o \in V\}
$$

По лемме 1 ,

$$
o_{i} \in J(\rho, q, V) \Longleftrightarrow t_{i}-t_{q} \in\left[F_{L}\left(x, y_{i}\right), F_{R}\left(x, y_{i}\right)\right] .
$$

Рассмотрим несколько случаев.

1. Пусть $(x, y)$ таковы, что $F_{R}(x, y) \leqslant 0$. Тогда по условию верно, что

$$
\begin{aligned}
& F_{L}(x, y)=\psi^{*}(y)+\psi_{1}^{*}(x), \\
& F_{R}(x, y)=\psi^{*}(y)+\psi_{2}^{*}(x),
\end{aligned}
$$

то есть

$$
\begin{aligned}
t_{i}-t_{q} \in\left[F_{L}\left(x, y_{i}\right), F_{R}\left(x, y_{i}\right)\right] & \Longleftrightarrow t_{i}-\psi^{*}\left(y_{i}\right) \in\left[t_{q}+\psi_{1}^{*}(x), t_{q}+\psi_{2}^{*}(x)\right] \\
& \Longleftrightarrow \varphi\left(o_{i}\right) \in\left[\varphi_{1}(q), \varphi_{2}(q)\right] \\
& \Longleftrightarrow \varphi\left(o_{i}\right) \in J\left(\left[\varphi_{1}(q), \varphi_{2}(q)\right], Z\right),
\end{aligned}
$$

где

$$
Z=\{\varphi(o): o \in V\}
$$

2. Пусть $(x, y)$ таковы, что $F_{L}(x, y) \leqslant 0, F_{R}(x, y)>0$. Тогда по доказанному ранее верно, что

$$
F_{L}(x, y)=\psi^{*}(y)+\psi_{1}^{*}(x), \quad \psi^{*}(y)+\psi_{2}^{*}(x)>0 .
$$

Поскольку $o_{i} \in V\left(t_{q}\right)$, то $t_{i}-t_{q} \leqslant 0$ и

$$
\begin{aligned}
t_{i}-t_{q} \in\left[F_{L}\left(x, y_{i}\right), F_{R}\left(x, y_{i}\right)\right] & \Longleftrightarrow t_{i}-t_{q} \in\left[F_{L}\left(x, y_{i}\right), \psi^{*}(y)+\psi_{2}^{*}(x)\right] \\
& \Longleftrightarrow t_{i}-t_{q} \in\left[\psi^{*}(y)+\psi_{1}^{*}(x), \psi^{*}(y)+\psi_{2}^{*}(x)\right] \\
& \Longleftrightarrow \varphi\left(o_{i}\right) \in\left[\varphi_{1}(q), \varphi_{2}(q)\right] \\
& \Longleftrightarrow \varphi\left(o_{i}\right) \in J\left(\left[\varphi_{1}(q), \varphi_{2}(q)\right], Z\right) .
\end{aligned}
$$

3. Пусть $(x, y)$ таковы, что $F_{L}(x, y)>0$. Тогда по доказанному ранее верно, что

$$
\psi^{*}(y)+\psi_{1}^{*}(x)>0, \quad \psi^{*}(y)+\psi_{2}^{*}(x)>0 .
$$

Поскольку $o_{i} \in V\left(t_{q}\right)$, то $t_{i}-t_{q} \leqslant 0$ и

$$
\begin{aligned}
t_{i}-t_{q} \notin\left[F_{L}\left(x, y_{i}\right), F_{R}\left(x, y_{i}\right)\right] & \Longleftrightarrow t_{i}-t_{q} \notin\left[\psi^{*}(y)+\psi_{2}^{*}(x), \psi^{*}(y)+\psi_{2}^{*}(x)\right] \\
& \Longleftrightarrow \varphi\left(o_{i}\right) \notin J\left(\left[\varphi_{1}(q), \varphi_{2}(q)\right], Z\right) .
\end{aligned}
$$

Достаточность доказана. 


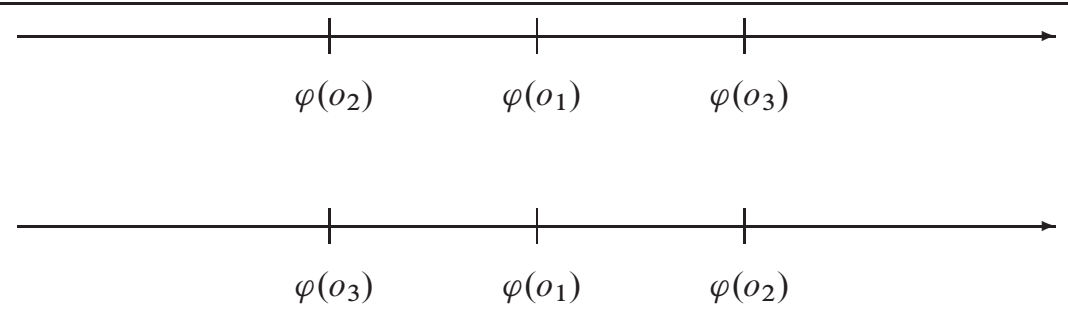

Рис. 3. Два возможных расположения точек $\varphi\left(o_{1}\right), \varphi\left(o_{2}\right), \varphi\left(o_{3}\right)$

\section{4. Необходимость}

Лемма 2. Если для задачи об опасной близости $\left(f, f_{q}, \rho\right)$ сущеествует четыре объекта $\left\{o_{1}, o_{2}, o_{3}, o_{4}\right\}$ и четыре запроса $\left\{q_{1}, q_{2}, q_{3}, q_{4}\right\}$, такие что

$$
\begin{aligned}
& V\left(t_{q}^{1}\right)=V\left(t_{q}^{2}\right)=V\left(t_{q}^{3}\right)=V\left(t_{q}^{4}\right)=\left\{o_{1}, o_{2}, o_{3}, o_{4}\right\}=V, \\
& J\left(\rho, q_{1}, V\right)=\left\{o_{1}, o_{2}\right\}, \quad J\left(\rho, q_{2}, V\right)=\left\{o_{1}, o_{3}\right\}, \\
& J\left(\rho, q_{3}, V\right)=\left\{o_{1}, o_{2}, o_{4}\right\}, \quad J\left(\rho, q_{4}, V\right)=\left\{o_{1}, o_{3}, o_{4}\right\},
\end{aligned}
$$

то задача об опасной близости $(f, f q, \rho)$ не может быть сведена к задаче одномерного интервального поиска.

Доказательство. Пусть утверждение леммы неверно. Тогда существуют отображения $\varphi, \varphi_{1}, \varphi_{2}: \mathbf{R} \times[0,1] \rightarrow \mathbf{R}$, такие, что для библиотеки $V$, любого запроса $q_{j}, j \in\{1,2,3,4\}$, и любого объекта $o_{i}, i \in\{1,2,3,4\}$ справедливо соотношение

$$
o_{i} \in J\left(\rho, q_{j}, V\right) \Longleftrightarrow \varphi\left(o_{i}\right) \in J\left(\left[\varphi_{1}\left(q_{j}\right), \varphi_{2}\left(q_{j}\right)\right], Z\right),
$$

где

$$
Z=\left\{\varphi\left(o_{i}\right): i \in\{1,2,3,4\}\right\}
$$

Так как

$$
J\left(\rho, q_{1}, V\right)=\left\{o_{1}, o_{2}\right\}
$$

на прямой $\mathbf{R}$ должен существовать отрезок $\left[\varphi_{1}\left(q_{1}\right), \varphi_{2}\left(q_{1}\right)\right]$, который содержит только точки $\varphi\left(o_{1}\right), \varphi\left(o_{2}\right)$ и не содержит точки $\varphi\left(o_{3}\right), \varphi\left(o_{4}\right)$.

Так как

$$
J\left(\rho, q_{2}, V\right)=\left\{o_{1}, o_{3}\right\}
$$

на прямой $\mathbf{R}$ должен существовать отрезок $\left[\varphi_{1}\left(q_{2}\right), \varphi_{2}\left(q_{2}\right)\right]$, который содержит только точки $\varphi\left(o_{1}\right), \varphi\left(o_{3}\right)$ и не содержит точки $\varphi\left(o_{2}\right), \varphi\left(o_{4}\right)$.

Отсюда следует, что возможны только два расположения тройки точек $\varphi\left(o_{1}\right), \varphi\left(o_{2}\right)$, $\varphi\left(o_{3}\right)$ (они показаны на рис. 3$)$, и при этом в обоих случаях $\varphi\left(o_{4}\right)$ не может лежать между $\varphi\left(o_{2}\right)$ и $\varphi\left(o_{3}\right)$.

Так как

$$
J\left(\rho, q_{3}, V\right)=\left\{o_{1}, o_{2}, o_{4}\right\}
$$




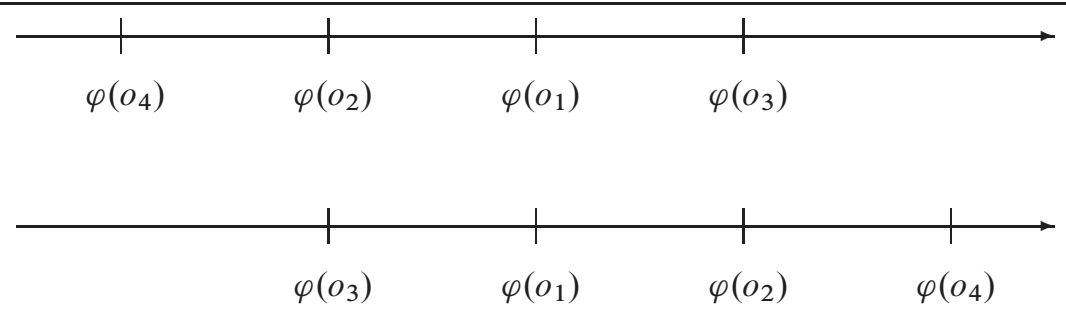

Рис. 4. Два возможных расположения точек $\varphi\left(o_{1}\right), \varphi\left(o_{2}\right), \varphi\left(o_{3}\right), \varphi\left(o_{4}\right)$

на прямой $\mathbf{R}$ должен существовать отрезок $\left[\varphi_{1}\left(q_{3}\right), \varphi_{2}\left(q_{3}\right)\right]$, который содержит только точки $\varphi\left(o_{1}\right), \varphi\left(o_{2}\right), \varphi\left(o_{4}\right)$ и не содержит точки $\varphi\left(o_{3}\right)$. Тогда в первом случае точка $\varphi\left(o_{4}\right)$ должна располагаться на прямой $\mathbf{R}$ правее $\varphi\left(o_{2}\right)$, а во втором случае - левее $\varphi\left(o_{2}\right)$. Данное расположение показано на рис. 4.

Но, так как

$$
J\left(\rho, q_{4}, V\right)=\left\{o_{1}, o_{3}, o_{4}\right\},
$$

на прямой $\mathbf{R}$ должен существовать отрезок $\left[\varphi_{1}\left(q_{4}\right), \varphi_{2}\left(q_{4}\right)\right]$, который содержит только точки $\varphi\left(o_{1}\right), \varphi\left(o_{3}\right), \varphi\left(o_{4}\right)$ и не содержит точки $\varphi\left(o_{2}\right)$. На рис. 4 видно, что такого отрезка не существует в обоих случаях.

Тем самым лемма 2 доказана.

Лемма 3. Если задача об опасной близости $\left(f, f_{q}, \rho\right)$ сводится к задаче одномерного интервального поиска, то существуют функции $\psi_{L}^{\prime}, \psi_{R}^{\prime}:[0,1] \rightarrow \mathbf{R} u$ функция $g$ : $[0,1]^{2} \rightarrow \mathbf{R}$, такие, что для любой пары $(x, y)$ такой, что $F_{R}(x, y) \leqslant 0$

$$
\begin{aligned}
& F_{L}(x, y)=g(x, y)+\psi_{L}^{\prime}(x), \\
& F_{R}(x, y)=g(x, y)+\psi_{R}^{\prime}(x) .
\end{aligned}
$$

Доказательство. Докажем утверждение для случая $F_{R}(x, y)<0$. Пусть

$$
\zeta(x, y)=F_{R}(x, y)-F_{L}(x, y) .
$$

Покажем, что $\zeta(x, y)$ зависит существенно только от $x$. Предположим, что это не так. Тогда существует тройка $\left(x_{0}, y^{1}, y^{2}\right)$, такая, что $F_{R}\left(x_{0}, y^{1}\right)<0, F_{R}\left(x_{0}, y^{2}\right)<0$ и $\zeta\left(x_{0}, y^{1}\right)>\zeta\left(x_{0}, y^{2}\right)$. Для доказательства будем использовать схему (17). Для объекта $o_{i}=\left(t_{i}, y^{i}\right)$ положим

$$
t_{i}^{\prime}=t_{i}-F_{R}\left(x_{0}, y^{i}\right) .
$$

Тогда для объекта $o_{i} \in V\left(t_{q}\right)$ и запроса $q\left(t_{q}, x_{0}\right)$, в соответствии с леммой 1 ,

$$
o_{i} \in J(\rho, q, V) \Longleftrightarrow t_{q} \in\left[t^{\prime}, t^{\prime}+\zeta\left(x_{0}, y^{i}\right)\right] .
$$

Выберем

$$
\begin{array}{r}
\varepsilon \in\left(0, \min \left\{\frac{\zeta\left(x_{0}, y^{1}\right)-\zeta\left(x_{0}, y^{2}\right)}{3}, \frac{\zeta\left(x_{0}, y^{1}\right)}{2}, \zeta\left(x_{0}, y^{2}\right),-\frac{F_{R}\left(x_{0}, y^{2}\right)}{2},-\frac{F_{R}\left(x_{0}, y^{1}\right)}{2},\right.\right. \\
\left.\left.\frac{1}{2}\left(F_{L}\left(x_{0}, y^{2}\right)+\tau_{\max }\right), \frac{1}{3}\left(\tau_{\max }+F_{R}\left(x_{0}, y^{1}\right)\right)\right\}\right)
\end{array}
$$




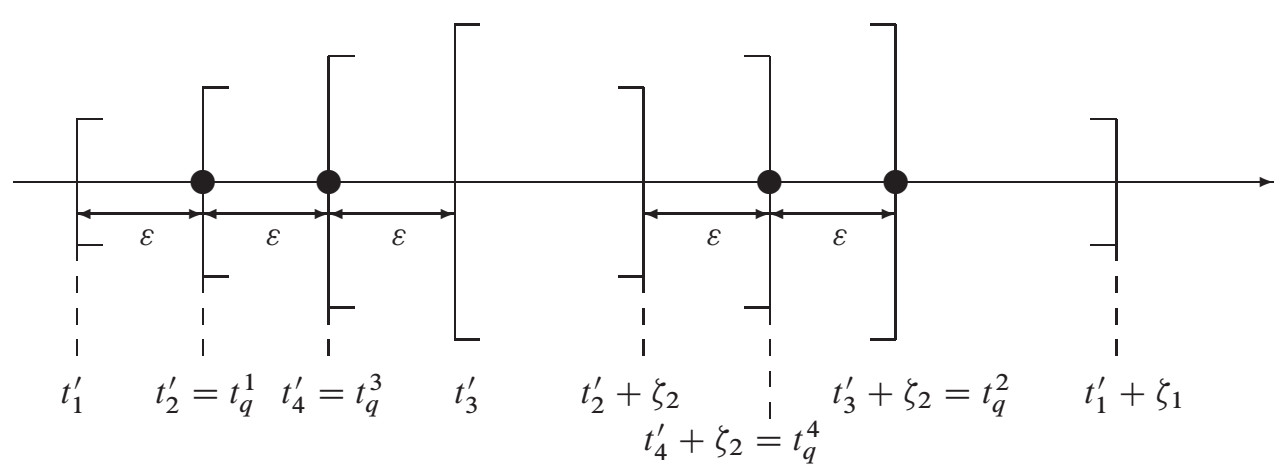

Рис. 5. Расположение моментов появления объектов и запросов

и произвольное число $t$. Зададим объекты следующим образом:

$$
\begin{array}{ll}
o_{1}=\left(t+F_{R}\left(x_{0}, y^{1}\right), y^{1}\right), & o_{2}=\left(t+F_{R}\left(x_{0}, y^{2}\right)+\varepsilon, y^{2}\right), \\
o_{3}=\left(t+F_{R}\left(x_{0}, y^{2}\right)+3 \varepsilon, y^{2}\right), & o_{4}=\left(t+F_{R}\left(x_{0}, y^{2}\right)+2 \varepsilon, y^{2}\right) .
\end{array}
$$

Для этих объектов

$$
t_{1}^{\prime}=t, \quad t_{2}^{\prime}=t+\varepsilon, \quad t_{3}^{\prime}=t+3 \varepsilon, \quad t_{4}^{\prime}=t+2 \varepsilon .
$$

Зададим запросы следующим образом:

$$
\begin{array}{ll}
q_{1}=\left(t+\varepsilon, x_{0}\right), & q_{2}=\left(t+3 \varepsilon+\zeta\left(x_{0}, y^{2}\right), x_{0}\right), \\
q_{3}=\left(t+2 \varepsilon, x_{0}\right), & q_{4}=\left(t+\zeta\left(x_{0}, y^{2}\right)+2 \varepsilon, x_{0}\right) .
\end{array}
$$

Моменты $t_{i}^{\prime}$ и $t_{q}^{j}$ показаны на рис. 5 , где $\zeta_{i}=\zeta\left(x_{0}, y^{i}\right)$.

Покажем, что

$$
V=V\left(t_{q}^{1}\right)=V\left(t_{q}^{2}\right)=V\left(t_{q}^{3}\right)=V\left(t_{q}^{4}\right)=\left\{o_{1}, o_{2}, o_{3}, o_{4}\right\},
$$

то есть $t_{q}^{j} \in\left[t_{i}, t_{i}+\tau_{\max }\right]$, где $i, j \in\{1,2,3,4\}$. Для этого необходимо показать, что $\min _{j=1, \ldots, 4} t_{q}^{j} \geqslant \max _{i=1, \ldots 4} t_{i}$ и $\max _{j=1, \ldots, 4} t_{q}^{j} \leqslant \min _{i=1, \ldots, 4} t_{i}+\tau_{\max }$.

Справедливы следующие утверждения:

$$
\min _{j=1, \ldots, 4} t_{q}^{j}=t+\varepsilon \geqslant t+\max _{k=1,2} F_{R}\left(x_{0}, y^{k}\right)+3 \varepsilon=\max _{i=1, \ldots, 4} t_{i}
$$

что равносильно $\varepsilon \leqslant-\max _{k=1,2} \frac{1}{2} F_{R}\left(x_{0}, y^{k}\right)$ и это верно из-за выбора $\varepsilon$;

$$
\begin{aligned}
\max _{j=1, \ldots, 4} t_{q}^{j} & =t+3 \varepsilon+\zeta\left(x_{0}, y^{2}\right) \geqslant \min _{i=1, \ldots, 4} t_{i}+\tau_{\max } \\
& =t+\min \left\{F_{R}\left(x_{0}, y^{1}\right), F_{R}\left(x_{0}, y^{2}\right)+\varepsilon\right\}+\tau_{\max }
\end{aligned}
$$


что выполнено, поскольку в силу выбора $\varepsilon$ справедливы неравенства

$$
\varepsilon \geqslant \frac{F_{L}\left(x_{0}, y^{2}\right)+\tau_{\max }}{2}, \quad \varepsilon \geqslant \frac{F_{R}\left(x_{0}, y^{1}\right)+\tau_{\max }}{3} .
$$

Используя утверждение леммы 1, покажем, что справедливы равенства

$$
\begin{array}{ll}
J\left(\rho, q_{1}, V\right)=\left\{o_{1}, o_{2}\right\}, & J\left(\rho, q_{2}, V\right)=\left\{o_{1}, o_{3}\right\}, \\
J\left(\rho, q_{3}, V\right)=\left\{o_{1}, o_{2}, o_{4}\right\}, & J\left(\rho, q_{4}, V\right)=\left\{o_{1}, o_{3}, o_{4}\right\} .
\end{array}
$$

Далее,

$$
o_{1} \in J\left(\rho, q_{1}, V\right) \Longleftrightarrow F_{R}\left(x_{0}, y^{1}\right)-\varepsilon \in\left[F_{L}\left(x_{0}, y^{1}\right), F_{R}\left(x_{0}, y^{1}\right)\right],
$$

что верно, поскольку в силу (18) $\varepsilon<F_{R}\left(x_{0}, y^{1}\right)-F_{L}\left(x_{0}, y^{1}\right)=\zeta\left(x_{0}, y^{1}\right)$;

$$
o_{2} \in J\left(\rho, q_{1}, V\right) \Longleftrightarrow F_{R}\left(x_{0}, y^{2}\right) \in\left[F_{L}\left(x_{0}, y^{2}\right), F_{R}\left(x_{0}, y^{2}\right)\right],
$$

что верно;

$$
o_{3} \notin J\left(\rho, q_{1}, V\right) \Longleftrightarrow F_{R}\left(x_{0}, y^{2}\right)+2 \varepsilon \notin\left[F_{L}\left(x_{0}, y^{2}\right), F_{R}\left(x_{0}, y^{2}\right)\right],
$$

что верно, поскольку $\varepsilon>0$;

$$
o_{4} \notin J\left(\rho, q_{1}, V\right) \Longleftrightarrow F_{R}\left(x_{0}, y^{2}\right)+\varepsilon \notin\left[F_{L}\left(x_{0}, y^{2}\right), F_{R}\left(x_{0}, y^{2}\right)\right],
$$

что верно, поскольку $\varepsilon>0$;

$$
\begin{aligned}
o_{1} \in J\left(\rho, q_{2}, V\right) & \Longleftrightarrow F_{R}\left(x_{0}, y^{1}\right)-3 \varepsilon-\zeta\left(x_{0}, y^{2}\right) \in\left[F_{L}\left(x_{0}, y^{1}\right), F_{R}\left(x_{0}, y^{1}\right)\right] \\
& \Longleftrightarrow-3 \varepsilon-\zeta\left(x_{0}, y^{2}\right) \in\left[-\zeta\left(x_{0}, y^{1}\right), 0\right] \\
& \Longleftrightarrow \varepsilon \in\left[0, \frac{1}{3}\left(\zeta\left(x_{0}, y^{1}\right)-\zeta\left(x_{0}, y^{2}\right)\right)\right]
\end{aligned}
$$

что верно по условию (18) выбора $\varepsilon$;

$$
o_{2} \notin J\left(\rho, q_{2}, V\right) \Longleftrightarrow F_{L}\left(x_{0}, y^{2}\right)-2 \varepsilon \notin\left[F_{L}\left(x_{0}, y^{2}\right), F_{R}\left(x_{0}, y^{2}\right)\right],
$$

что верно, поскольку $\varepsilon>0$;

$$
o_{3} \in J\left(\rho, q_{2}, V\right) \Longleftrightarrow F_{L}\left(x_{0}, y^{2}\right) \in\left[F_{L}\left(x_{0}, y^{2}\right), F_{R}\left(x_{0}, y^{2}\right)\right],
$$

что верно;

$$
o_{4} \notin J\left(\rho, q_{2}, V\right) \Longleftrightarrow F_{L}\left(x_{0}, y^{2}\right)-\varepsilon \notin\left[F_{L}\left(x_{0}, y^{2}\right), F_{R}\left(x_{0}, y^{2}\right)\right],
$$

что верно, поскольку $\varepsilon>0$;

$$
o_{1} \in J\left(\rho, q_{3}, V\right) \Longleftrightarrow F_{R}\left(x_{0}, y^{1}\right)-2 \varepsilon \in\left[F_{L}\left(x_{0}, y^{1}\right), F_{R}\left(x_{0}, y^{1}\right)\right],
$$

что верно, поскольку $0<\varepsilon<\zeta\left(x_{0}, y^{1}\right) / 2$ в силу (18);

$$
o_{2} \in J\left(\rho, q_{3}, V\right) \Longleftrightarrow F_{R}\left(x_{0}, y^{2}\right)-\varepsilon \in\left[F_{L}\left(x_{0}, y^{2}\right), F_{R}\left(x_{0}, y^{2}\right)\right],
$$


что верно, поскольку $0<\varepsilon<\zeta\left(x_{0}, y^{2}\right)$ в силу (18);

$$
o_{3} \notin J\left(\rho, q_{3}, V\right) \Longleftrightarrow F_{R}\left(x_{0}, y^{2}\right)+\varepsilon \notin\left[F_{L}\left(x_{0}, y^{2}\right), F_{R}\left(x_{0}, y^{2}\right)\right],
$$

что верно, поскольку $\varepsilon>0$;

$$
o_{4} \in J\left(\rho, q_{3}, V\right) \Longleftrightarrow F_{R}\left(x_{0}, y^{2}\right) \in\left[F_{L}\left(x_{0}, y^{2}\right), F_{R}\left(x_{0}, y^{2}\right)\right],
$$

что верно;

$$
o_{1} \in J\left(\rho, q_{4}, V\right) \Longleftrightarrow F_{R}\left(x_{0}, y^{1}\right)-2 \varepsilon-\zeta\left(x_{0}, y^{2}\right) \in\left[F_{L}\left(x_{0}, y^{1}\right), F_{R}\left(x_{0}, y^{1}\right)\right],
$$

что верно, поскольку $0<2 \varepsilon<\zeta\left(x_{0}, y^{1}\right)-\zeta\left(x_{0}, y^{2}\right)$ в силу (18);

$$
o_{2} \notin J\left(\rho, q_{4}, V\right) \Longleftrightarrow F_{L}\left(x_{0}, y^{2}\right)-\varepsilon \notin\left[F_{L}\left(x_{0}, y^{2}\right), F_{R}\left(x_{0}, y^{2}\right)\right],
$$

что верно, поскольку $\varepsilon>0$;

$$
o_{3} \in J\left(\rho, q_{4}, V\right) \Longleftrightarrow F_{L}\left(x_{0}, y^{2}\right)+\varepsilon \in\left[F_{L}\left(x_{0}, y^{2}\right), F_{R}\left(x_{0}, y^{2}\right)\right],
$$

что верно, поскольку $0<\varepsilon<F_{R}\left(x_{0}, y^{2}\right)-F_{L}\left(x_{0}, y^{2}\right)=\zeta\left(x_{0}, y^{2}\right)$;

$$
o_{4} \in J\left(\rho, q_{4}, V\right) \Longleftrightarrow F_{L}\left(x_{0}, y^{2}\right) \in\left[F_{L}\left(x_{0}, y^{2}\right), F_{R}\left(x_{0}, y^{2}\right)\right],
$$

что верно.

Докажем теперь утверждение для случая $F_{R}(x, y)=0$. Выберем последовательность $\left(x_{n}\right) \rightarrow x$ такую, что $x_{n}>x$. Для этой последовательности получим, что $F_{R}\left(x_{n}, y\right)<0$, $F_{R}\left(x_{n}, y\right) \rightarrow F_{R}(x, y), F_{L}\left(x_{n}, y\right) \rightarrow F_{L}(x, y)$. По доказанному, $F_{R}\left(x_{n}, y\right)-F_{L}\left(x_{n}, y\right)$ зависит только от $x_{n}$. Обозначим эту функцию $\psi\left(x_{n}\right)$. Данная функция непрерывна, так как непрерывны функции $F_{R}\left(x_{n}, y\right), F_{L}\left(x_{n}, y\right)$. Поэтому разность $F_{R}(x, y)-F_{L}(x, y)=\psi(x)$ также не зависит от $y$.

Лемма 3 доказана.

Введем обозначение

$$
h_{x_{0}, x_{1}}(y)=F_{L}\left(x_{0}, y\right)-F_{L}\left(x_{1}, y\right) .
$$

Лемма 4. Если задача об опасной близости $\left(f, f_{q}, \rho\right)$ сводится к задаче одномерного интервального поиска, то для любых $x_{0}, x_{1} \in[0,1]$ таких, что $x_{0}<x_{1}$, функиия $h_{x_{0}, x_{1}}(y)$ принимает не более двух значений для у из области

$$
F_{L}\left(x_{0}, y\right)<0, \quad h_{x_{0}, x_{1}}(y) \leqslant F_{L}(1,0)+\tau_{\max } .
$$

Доказательство. Сначала заметим, что $h_{x_{0}, x_{1}}(y)>0$ для любого $y$ при $x_{0}<x_{1}$.

Предположим, что утверждение леммы неверно, то есть существуют $y^{0}, y^{1}, y^{2} \in[0,1]$ из области (19), такие, что

$$
0<h_{x_{0}, x_{1}}\left(y^{0}\right)<h_{x_{0}, x_{1}}\left(y^{1}\right)<h_{x_{0}, x_{1}}\left(y^{2}\right) .
$$

Будем использовать схему доказательства (17).

Выберем

$$
\begin{aligned}
& \varepsilon \in\left(0, \min \left\{h_{x_{0}, x_{1}}\left(y^{1}\right), h_{x_{0}, x_{1}}\left(y^{2}\right)-h_{x_{0}, x_{1}}\left(y^{1}\right), h_{x_{0}, x_{1}}\left(y^{1}\right)-h_{x_{0}, x_{1}}\left(y^{0}\right),\right.\right. \\
& \left.\left.-F_{L}\left(x_{0}, y^{0}\right),-F_{L}\left(x_{0}, y^{1}\right), F_{L}\left(x_{1}, y^{1}\right)+\tau_{\max }, \min _{\substack{i=0,1 \\
j=0,1,2}}\left\{F_{R}\left(x_{i}, y^{j}\right)-F_{L}\left(x_{i}, y^{j}\right)\right\}\right\}\right) .
\end{aligned}
$$


Введем обозначение

$$
t_{q}^{2}=F_{L}\left(x_{0}, y^{1}\right)-F_{L}\left(x_{1}, y^{1}\right)=h_{x_{0}, x_{1}}\left(y^{1}\right) .
$$

Заметим, что $t_{q}^{2}>\varepsilon>0$ согласно (21).

Зададим параметры объектов и запросов следующим образом:

$$
\begin{array}{rlrl}
o_{1}=\left(t_{1}, y_{1}\right)=\left(F_{L}\left(x_{0}, y^{1}\right), y^{1}\right), & o_{2} & =\left(t_{2}, y_{2}\right)=\left(F_{L}\left(x_{0}, y^{0}\right), y^{0}\right), \\
o_{3}=\left(t_{3}, y_{3}\right)=\left(F_{L}\left(x_{1}, y^{2}\right)+t_{q}^{2}, y^{2}\right), & o_{4} & =\left(t_{4}, y_{4}\right)=\left(F_{L}\left(x_{1}, y^{1}\right)+t_{q}^{2}-\varepsilon, y^{1}\right) \\
& =\left(F_{L}\left(x_{0}, y^{1}\right)-\varepsilon, y^{1}\right), \\
q_{1}=\left(t_{q}^{1}, x^{1}\right)=\left(0, x_{0}\right), & q_{2}=\left(t_{q}^{2}, x^{2}\right)=\left(t_{q}^{2}, x_{1}\right), \\
q_{3}=\left(t_{q}^{3}, x^{3}\right)=\left(-\varepsilon, x_{0}\right), & q_{4}=\left(t_{q}^{4}, x^{4}\right)=\left(t_{q}^{2}-\varepsilon, x_{1}\right) .
\end{array}
$$

Покажем, что

$$
V\left(t_{q}^{1}\right)=V\left(t_{q}^{2}\right)=V\left(t_{q}^{3}\right)=V\left(t_{q}^{4}\right)=\left\{o_{1}, o_{2}, o_{3}, o_{4}\right\}
$$

По определению, $o=(t, y) \in V\left(t_{q}\right)$, если $t_{q} \in\left[t, t+\tau_{\mathrm{max}}\right]$. Таким образом, необходимо показать, что

$$
\begin{aligned}
& \min _{1 \leqslant j \leqslant 4} t_{q}^{j} \geqslant \max _{1 \leqslant i \leqslant 4} t_{i}, \\
& \max _{1 \leqslant j \leqslant 4} t_{q}^{j} \leqslant \min _{1 \leqslant i \leqslant 4} t_{i}+\tau_{\max } .
\end{aligned}
$$

Разобьем доказательство на две части.

Часть 1. Поскольку

$$
\varepsilon \leqslant \min \left\{-F_{L}\left(x_{0}, y^{0}\right),-F_{L}\left(x_{0}, y^{1}\right)\right\}
$$

в силу (21) верно

$$
\begin{aligned}
\min _{1 \leqslant j \leqslant 4} t_{q}^{j} \geqslant \max _{1 \leqslant i \leqslant 4} t_{i} & \Longleftrightarrow-\varepsilon \geqslant \max \left\{F_{L}\left(x_{0}, y^{0}\right), F_{L}\left(x_{0}, y^{1}, F_{L}\left(x_{1}, y^{2}\right)+t_{q}^{2}\right)\right\} \\
& \Longleftrightarrow \varepsilon \leqslant \min \left\{-F_{L}\left(x_{0}, y^{0}\right),-F_{L}\left(x_{0}, y^{1}\right),-F_{L}\left(x_{1}, y^{2}\right)-h_{x_{0}, x_{1}}\left(y^{1}\right)\right\},
\end{aligned}
$$

где последнее неравенство вытекает из соотношений

$$
\begin{aligned}
\varepsilon<h_{x_{0}, x_{1}}\left(y^{2}\right)-h_{x_{0}, x_{1}}\left(y^{1}\right) & =F_{L}\left(x_{0}, y^{2}\right)-F_{L}\left(x_{1}, y^{2}\right)-h_{x_{0}, x_{1}}\left(y^{1}\right) \\
& <-F_{L}\left(x_{1}, y^{2}\right)-h_{x_{0}, x_{1}}\left(y^{1}\right)
\end{aligned}
$$

последнее верно, поскольку $F_{L}\left(x_{0}, y^{2}\right)<0$.

Часть 2. Верно, что

$$
\begin{aligned}
\max _{j} t_{q}^{j} \leqslant \min _{i} t_{i}+\tau_{\max } \Longleftrightarrow t_{q}^{2} & =F_{L}\left(x_{0}, y^{1}\right)-F_{L}\left(x_{1}, y^{1}\right) \\
& \leqslant \min \left\{t_{1}, t_{2}, t_{3}, t_{4}\right\}+\tau_{\max } \\
& =\min \left\{F_{L}\left(x_{0}, y^{0}\right), t_{q}^{2}+F_{L}\left(x_{1}, y^{2}\right), F_{L}\left(x_{0}, y^{1}\right)-\varepsilon\right\}+\tau_{\max } .
\end{aligned}
$$


Проверим каждое из этих неравенств:

$$
F_{L}\left(x_{0}, y^{1}\right)-F_{L}\left(x_{1}, y^{1}\right) \leqslant F_{L}\left(x_{0}, y^{0}\right)+\tau_{\max },
$$

так как по условию (19) леммы 4

$$
F_{L}\left(x_{0}, y^{1}\right)-F_{L}\left(x_{1}, y^{1}\right) \leqslant F_{L}(1,0)+\tau_{\max } \leqslant F_{L}\left(x_{0}, y^{0}\right)+\tau_{\max } ;
$$

далее,

$$
t_{q}^{2} \leqslant t_{q}^{2}+F_{L}\left(x_{1}, y^{2}\right)+\tau_{\max }
$$

так как $-F_{L}\left(x_{1}, y^{2}\right) \leqslant \tau_{\max }$ по свойству 3 ; наконец,

$$
F_{L}\left(x_{0}, y^{1}\right)-F_{L}\left(x_{1}, y^{1}\right) \leqslant F_{L}\left(x_{0}, y^{1}\right)-\varepsilon+\tau_{\max },
$$

так как $\varepsilon<F_{L}\left(x_{1}, y^{1}\right)+\tau_{\max }$ согласно (21).

Теперь, используя утверждение леммы 1 , покажем, что

$$
\begin{array}{ll}
J\left(\rho, q_{1}, V\right)=\left\{o_{1}, o_{2}\right\}, & J\left(\rho, q_{2}, V\right)=\left\{o_{1}, o_{3}\right\}, \\
J\left(\rho, q_{3}, V\right)=\left\{o_{1}, o_{2}, o_{4}\right\}, & J\left(\rho, q_{4}, V\right)=\left\{o_{1}, o_{3}, o_{4}\right\},
\end{array}
$$

для чего рассмотрим следующие шаги:

$$
\begin{aligned}
o_{1} \in J\left(\rho, q_{1}, V\right) & \Longleftrightarrow t_{1}-0 \in\left[F_{L}\left(x_{0}, y^{1}\right), F_{R}\left(x_{0}, y^{1}\right)\right] \\
& \Longleftrightarrow F_{L}\left(x_{0}, y^{1}\right) \in\left[F_{L}\left(x_{0}, y^{1}\right), F_{R}\left(x_{0}, y^{1}\right)\right],
\end{aligned}
$$

что верно;

$$
\begin{aligned}
o_{2} \in J\left(\rho, q_{1}, V\right) & \Longleftrightarrow t_{2}-0 \in\left[F_{L}\left(x_{0}, y^{0}\right), F_{R}\left(x_{0}, y^{0}\right)\right] \\
& \Longleftrightarrow F_{L}\left(x_{0}, y^{0}\right) \in\left[F_{L}\left(x_{0}, y^{0}\right), F_{R}\left(x_{0}, y^{0}\right)\right],
\end{aligned}
$$

что верно;

$$
\begin{aligned}
o_{3} \notin J\left(\rho, q_{1}, V\right) & \Longleftrightarrow t_{3}-0 \notin\left[F_{L}\left(x_{0}, y^{2}\right), F_{R}\left(x_{0}, y^{2}\right)\right] \\
& \Longleftrightarrow F_{L}\left(x_{1}, y^{2}\right)+F_{L}\left(x_{0}, y^{1}\right)-F_{L}\left(x_{1}, y^{1}\right) \notin\left[F_{L}\left(x_{0}, y^{2}\right), F_{R}\left(x_{0}, y^{2}\right)\right],
\end{aligned}
$$

что верно, поскольку по предположению (20) $h_{x_{0}, x_{1}}\left(y^{1}\right)<h_{x_{0}, x_{1}}\left(y^{2}\right)$, что равносильно

$$
F_{L}\left(x_{1}, y^{2}\right)+F_{L}\left(x_{0}, y^{1}\right)-F_{L}\left(x_{1}, y^{1}\right)<F_{L}\left(x_{0}, y^{2}\right)
$$

далее,

$$
\begin{aligned}
o_{4} \notin J\left(\rho, q_{1}, V\right) & \Longleftrightarrow t_{4}-0 \notin\left[F_{L}\left(x_{0}, y^{1}\right), F_{R}\left(x_{0}, y^{1}\right)\right] \\
& \Longleftrightarrow F_{L}\left(x_{0}, y^{1}\right)-\varepsilon \notin\left[F_{L}\left(x_{0}, y^{1}\right), F_{R}\left(x_{0}, y^{1}\right)\right],
\end{aligned}
$$

что верно;

$$
\begin{aligned}
o_{1} \in J\left(\rho, q_{2}, V\right) & \Longleftrightarrow t_{1}-t_{q}^{2} \in\left[F_{L}\left(x_{1}, y^{1}\right), F_{R}\left(x_{1}, y^{1}\right)\right] \\
& \Longleftrightarrow F_{L}\left(x_{1}, y^{1}\right) \in\left[F_{L}\left(x_{1}, y^{1}\right), F_{R}\left(x_{1}, y^{1}\right)\right],
\end{aligned}
$$


что верно;

$$
\begin{aligned}
o_{2} \notin J\left(\rho, q_{2}, V\right) & \Longleftrightarrow t_{2}-t_{q}^{2} \notin\left[F_{L}\left(x_{1}, y^{0}\right), F_{R}\left(x_{1}, y^{0}\right)\right] \\
& \Longleftrightarrow F_{L}\left(x_{0}, y^{0}\right)-F_{L}\left(x_{0}, y^{1}\right)+F_{L}\left(x_{1}, y^{1}\right) \notin\left[F_{L}\left(x_{1}, y^{0}\right), F_{R}\left(x_{1}, y^{0}\right)\right],
\end{aligned}
$$

что верно, так как по предположению (20) $h_{x_{0}, x_{1}}\left(y^{0}\right)<h_{x_{0}, x_{1}}\left(y^{1}\right)$, что равносильно

$$
F_{L}\left(x_{0}, y^{0}\right)-F_{L}\left(x_{0}, y^{1}\right)+F_{L}\left(x_{1}, y^{1}\right)<F_{L}\left(x_{1}, y^{0}\right)
$$

далее,

$$
\begin{aligned}
o_{3} \in J\left(\rho, q_{2}, V\right) & \Longleftrightarrow t_{3}-t_{q}^{2} \in\left[F_{L}\left(x_{1}, y^{2}\right), F_{R}\left(x_{1}, y^{2}\right)\right] \\
& \Longleftrightarrow F_{L}\left(x_{1}, y^{2}\right) \in\left[F_{L}\left(x_{1}, y^{2}\right), F_{R}\left(x_{1}, y^{2}\right)\right],
\end{aligned}
$$

что верно;

$$
\begin{aligned}
o_{4} \notin J\left(\rho, q_{2}, V\right) & \Longleftrightarrow t_{4}-t_{q}^{2} \notin\left[F_{L}\left(x_{1}, y^{1}\right), F_{R}\left(x_{1}, y^{1}\right)\right] \\
& \Longleftrightarrow F_{L}\left(x_{0}, y^{1}\right)-\varepsilon \notin\left[F_{L}\left(x_{1}, y^{1}\right), F_{R}\left(x_{1}, y^{1}\right)\right],
\end{aligned}
$$

что верно;

$$
\begin{aligned}
o_{1} \in J\left(\rho, q_{3}, V\right) & \Longleftrightarrow t_{1}+\varepsilon \in\left[F_{L}\left(x_{0}, y^{1}\right), F_{R}\left(x_{0}, y^{1}\right)\right] \\
& \Longleftrightarrow F_{L}\left(x_{0}, y^{1}\right)+\varepsilon \in\left[F_{L}\left(x_{0}, y^{1}\right), F_{R}\left(x_{0}, y^{1}\right)\right],
\end{aligned}
$$

что верно, поскольку $\varepsilon<F_{R}\left(x_{0}, y^{1}\right)-F_{L}\left(x_{0}, y^{1}\right)$ согласно (21);

$$
\begin{aligned}
o_{2} \in J\left(\rho, q_{3}, V\right) & \Longleftrightarrow t_{2}+\varepsilon \in\left[F_{L}\left(x_{0}, y^{0}\right), F_{R}\left(x_{0}, y^{0}\right)\right] \\
& \Longleftrightarrow F_{L}\left(x_{0}, y^{0}\right)+\varepsilon \in\left[F_{L}\left(x_{0}, y^{0}\right), F_{R}\left(x_{0}, y^{0}\right)\right],
\end{aligned}
$$

что верно, поскольку $\varepsilon<F_{R}\left(x_{0}, y^{0}\right)-F_{L}\left(x_{0}, y^{0}\right)$ согласно (21);

$$
\begin{aligned}
o_{3} \notin J\left(\rho, q_{3}, V\right) \Longleftrightarrow & t_{3}+\varepsilon \notin\left[F_{L}\left(x_{0}, y^{2}\right), F_{R}\left(x_{0}, y^{2}\right)\right] \\
\Longleftrightarrow & F_{L}\left(x_{1}, y^{2}\right)+F_{L}\left(x_{0}, y^{1}\right)-F_{L}\left(x_{1}, y^{1}\right) \\
& +\varepsilon \notin\left[F_{L}\left(x_{0}, y^{2}\right), F_{R}\left(x_{0}, y^{2}\right)\right],
\end{aligned}
$$

что верно, поскольку $\varepsilon<h_{x_{0}, x_{1}}\left(y^{2}\right)-h_{x_{0}, x_{1}}\left(y^{1}\right)$, а это неравенство равносильно неравенству

$$
F_{L}\left(x_{1}, y^{2}\right)+F_{L}\left(x_{0}, y^{1}\right)-F_{L}\left(x_{1}, y^{1}\right)+\varepsilon<F_{L}\left(x_{0}, y^{2}\right)
$$

далее,

$$
\begin{aligned}
o_{4} \in J\left(\rho, q_{3}, V\right) & \Longleftrightarrow t_{4}+\varepsilon \in\left[F_{L}\left(x_{0}, y^{1}\right), F_{R}\left(x_{0}, y^{1}\right)\right] \\
& \Longleftrightarrow F_{L}\left(x_{0}, y^{1}\right) \in\left[F_{L}\left(x_{0}, y^{1}\right), F_{R}\left(x_{0}, y^{1}\right)\right],
\end{aligned}
$$

что верно;

$$
\begin{aligned}
o_{1} \in J\left(\rho, q_{4}, V\right) & \Longleftrightarrow t_{1}-t_{q}^{2}+\varepsilon \in\left[F_{L}\left(x_{1}, y^{1}\right), F_{R}\left(x_{1}, y^{1}\right)\right] \\
& \Longleftrightarrow F_{L}\left(x_{1}, y^{1}\right)+\varepsilon \in\left[F_{L}\left(x_{1}, y^{1}\right), F_{R}\left(x_{1}, y^{1}\right)\right],
\end{aligned}
$$




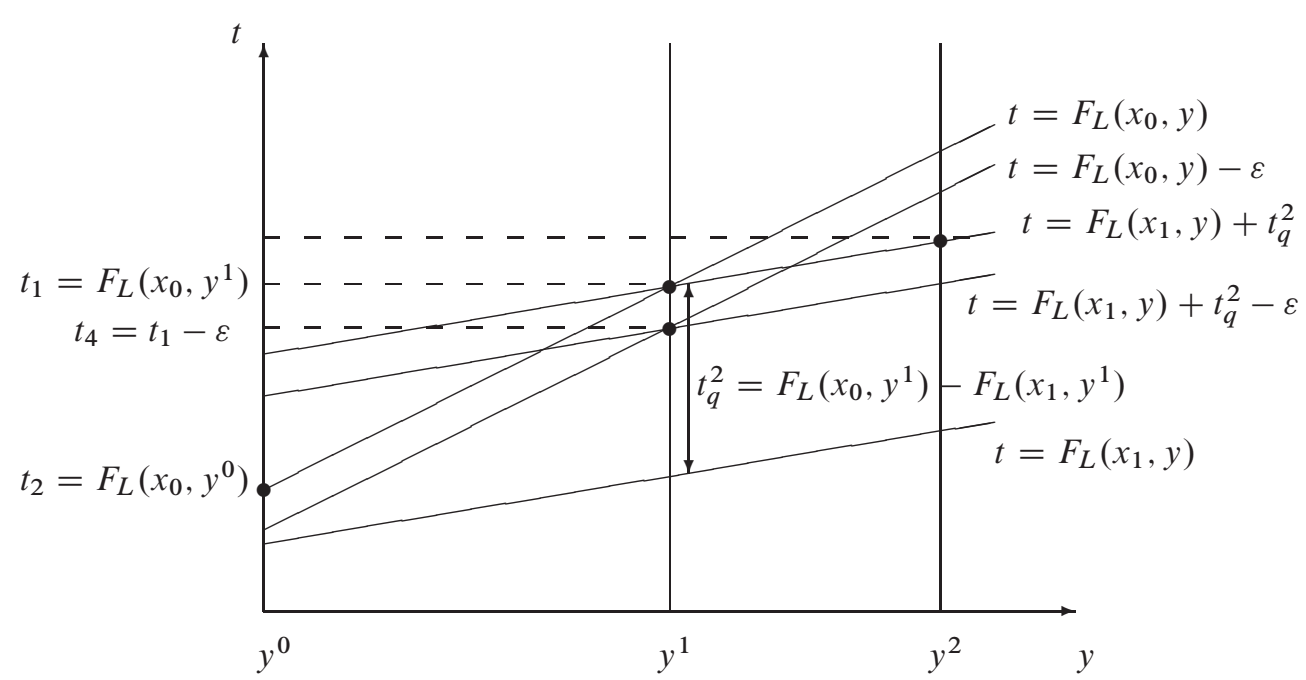

Рис. 6. Расположение моментов появления объектов и запросов для случая $y^{0}<y^{1}<y^{2}$

что верно, поскольку $\varepsilon<F_{R}\left(x_{1}, y^{1}\right)-F_{L}\left(x_{1}, y^{1}\right)$ согласно (21);

$$
\begin{gathered}
o_{2} \notin J\left(\rho, q_{4}, V\right) \Longleftrightarrow t_{2}-t_{q}^{2}+\varepsilon \notin\left[F_{L}\left(x_{1}, y^{0}\right), F_{R}\left(x_{1}, y^{0}\right)\right] \\
\Longleftrightarrow F_{L}\left(x_{0}, y^{0}\right)-F_{L}\left(x_{0}, y^{1}\right)+F_{L}\left(x_{1}, y^{1}\right) \\
\quad+\varepsilon \notin\left[F_{L}\left(x_{1}, y^{0}\right), F_{R}\left(x_{1}, y^{0}\right)\right],
\end{gathered}
$$

что верно, поскольку согласно $(21) \varepsilon<h_{x_{0}, x_{1}}\left(y^{2}\right)-h_{x_{0}, x_{1}}\left(y^{1}\right)$, что равносильно тому, что

$$
F_{L}\left(x_{0}, y^{0}\right)-F_{L}\left(x_{0}, y^{1}\right)+F_{L}\left(x_{1}, y^{1}\right)+\varepsilon<F_{L}\left(x_{1}, y^{0}\right)
$$

далее,

$$
\begin{aligned}
o_{3} \in J\left(\rho, q_{4}, V\right) & \Longleftrightarrow t_{3}-t_{q}^{2}+\varepsilon \in\left[F_{L}\left(x_{1}, y^{2}\right), F_{R}\left(x_{1}, y^{2}\right)\right] \\
& \Longleftrightarrow F_{L}\left(x_{1}, y^{2}\right)+\varepsilon \in\left[F_{L}\left(x_{1}, y^{2}\right), F_{R}\left(x_{1}, y^{2}\right)\right],
\end{aligned}
$$

что верно, поскольку $\varepsilon<F_{R}\left(x_{1}, y^{2}\right)-F_{L}\left(x_{1}, y^{2}\right)$ согласно (21);

$$
\begin{aligned}
o_{4} \in J\left(\rho, q_{4}, V\right) & \Longleftrightarrow t_{4}-t_{q}^{2}+\varepsilon \in\left[F_{L}\left(x_{1}, y^{1}\right), F_{R}\left(x_{1}, y^{1}\right)\right] \\
& \Longleftrightarrow F_{L}\left(x_{1}, y^{1}\right) \in\left[F_{L}\left(x_{1}, y^{1}\right), F_{R}\left(x_{1}, y^{1}\right)\right],
\end{aligned}
$$

что верно.

Отсюда, согласно лемме 2, следует справедливость утверждения леммы 4.

Рассмотрим теперь графическую интерпретацию доказательства леммы 4. Вспомним, что по основной лемме 1 объект $o_{i}=\left(t_{i}, y_{i}\right)$ из библиотеки $V=V\left(t_{q}\right)$ принадлежит ответу на запрос $q=\left(t_{q}, x\right)$ (то есть $\left.o_{i} \in J(\rho, q, V)\right)$ тогда и только тогда, когда $t_{i}-t_{q} \in\left[F_{L}\left(x, y_{i}\right), F_{R}\left(x, y_{i}\right)\right]$. Рассмотрим двумерный график на рис. 6 . На оси абсцисс будем откладывать координаты появления объектов, а на оси ординат - моменты появления объектов. Таким образом, каждому объекту $o_{i}=\left(t_{i}, y_{i}\right), i \in\{1,2,3,4\}$, будет 
соответствовать точка с координатой $y_{i}$ по оси абсцисс и координатой $t_{i}$ по оси ординат. На рис. 6 изображены данные точки для случая $y_{0}<y_{1}<y_{2}$.

Каждому запросу $\left(t_{q}, x^{0}\right)$ в данных координатах поставим в соответствие две функции

$$
\begin{aligned}
& t_{L}(y)=t_{q}+F_{L}\left(x^{0}, y\right), \\
& t_{R}(y)=t_{q}+F_{R}\left(x^{0}, y\right) .
\end{aligned}
$$

Таким образом, по основной лемме в ответ на запрос $\left(t_{q}, x^{0}\right)$ попадут те объекты из библиотеки $V=V\left(t_{q}\right)$, которым соответствуют точки, лежащие между линиями $t_{1}(y)$ и $t_{2}(y)$ (либо на самих линиях).

На рис. 6 для удобства изображены только нижние функции, соответствующие запросам $q_{1}, q_{2}, q_{3}$ и $q_{4}$, в виде прямых. Из рис. 6 видно, что

$$
\begin{array}{ll}
J\left(\rho, q_{1}, V\right)=\left\{o_{1}, o_{2}\right\}, & J\left(\rho, q_{2}, V\right)=\left\{o_{1}, o_{3}\right\}, \\
J\left(\rho, q_{3}, V\right)=\left\{o_{1}, o_{2}, o_{4}\right\}, & J\left(\rho, q_{4}, V\right)=\left\{o_{1}, o_{3}, o_{4}\right\} .
\end{array}
$$

Введем обозначение

$$
\Delta=F_{L}(1,0)+\tau_{\max } .
$$

Лемма 5. Существует число $\delta>0$, такое, что для любых $x, y \in[0,1]$

$$
F_{L}(x, y)-F_{L}(x+\delta, y)<\Delta .
$$

Справедливость данной леммы следует из непрерывности $F_{L}(x, y)$ по $x$ и по $y$ внутри квадрата $[-\rho, 1+\rho]^{2}$.

Лемма 6. Если $\delta$ - число, существование которого доказывается леммой 5, то для любой пары $(x, y) \in[0,1]^{2}$ такой, что $F_{L}(x, y) \leqslant 0$, и для любого $\delta_{0} \in(0, \delta)$ справедливо равенство

$$
h_{x, x+\delta_{0}}(y)=h_{x, x+\delta_{0}}(0) \text {. }
$$

Доказательство. Сначала докажем, что утверждение верно для пары $\left(x_{1}, y_{1}\right)$ такой, что $F_{L}\left(x_{1}, y_{1}\right)<0$.

Для $y_{1}=0$ утверждение очевидно. Пусть $y_{1}>0$.

Допустим противное, пусть $h_{x, x+\delta_{0}}\left(y_{1}\right) \neq h_{x, x+\delta_{0}}(0)$.

Поскольку $\delta_{0} \in(0, \delta)$, с использованием леммы 5 получаем

$$
h_{x, x+\delta_{0}}(y)=F_{L}\left(x_{1}, y\right)-F_{L}\left(x_{1}+\delta_{0}, y\right)<\Delta
$$

и, значит, по лемме $4 h_{x, x+\delta_{0}}(y)$ на отрезке $[0,1]$ может принимать не более двух различных значений. По предположению, одно из этих значений равно $h_{x, x+\delta_{0}}\left(y_{1}\right)$, а другое равно $h_{x, x+\delta_{0}}(0)$.

Построим две бесконечные последовательности $\left(y_{n}^{0}\right)$ и $\left(y_{n}^{1}\right)$, такие, что $h_{x, x+\delta_{0}}\left(y_{n}^{0}\right)=h_{x, x+\delta_{0}}(0), h_{x, x+\delta_{0}}\left(y_{n}^{1}\right)=h_{x, x+\delta_{0}}\left(y_{1}\right)$.

На первом шаге отнесем к первой последовательности 0 , а ко второй $y_{1}$, то есть $y_{1}^{0}=0, y_{1}^{1}=y_{1}$.

На втором шаге рассмотрим $y_{2}=\left|y_{1}^{0}-y_{1}^{1}\right| / 2$. Если $h_{x, x+\delta_{0}}\left(y_{2}\right)=h_{x, x+\delta_{0}}(0)$, то $y_{2}^{0}=y_{2}, y_{2}^{1}=y_{1}$, а если $h_{x, x+\delta_{0}}\left(y_{2}\right)=h_{x, x+\delta_{0}}\left(y_{1}\right)$, то $y_{2}^{0}=y_{0}, y_{2}^{1}=y_{2}$.

На $k$-м шаге рассмотрим $y_{k}=\left|y_{k-1}^{0}-y_{k-1}^{1}\right| / 2$. Если $h_{x, x+\delta_{0}}\left(y_{k}\right)=h_{x, x+\delta_{0}}(0)$, то $y_{k}^{0}=y_{k}, y_{k}^{1}=y_{k-1}^{1}$, а если $h_{x, x+\delta_{0}}\left(y_{k}\right)=h_{x, x+\delta_{0}}\left(y_{1}\right)$, то $y_{k}^{0}=y_{k-1}^{0}, y_{k}^{1}=y_{k}$. 
Таким образом, получаем, что все три последовательности $\left(y_{n}^{0}\right),\left(y_{n}^{1}\right)$ и $\left(y_{n}^{0}\right) \cup\left(y_{n}^{1}\right)$ имеют единственную предельную точку, одинаковую для всех трех последовательностей, то есть все три последовательности сходятся к одному пределу. Обозначим этот предел $y_{*}$.

Функция $h_{x, x+\delta_{0}}(y)$ непрерывна и

$$
h_{x, x+\delta_{0}}\left(y_{*}\right)=h_{x, x+\delta_{0}}\left(\lim _{n \rightarrow \infty} y_{n}^{0}\right)=\lim _{n \rightarrow \infty} h_{x, x+\delta_{0}}\left(y_{n}^{0}\right)=h_{x, x+\delta_{0}}(0) .
$$

Аналогично получаем, что

$$
h_{x, x+\delta_{0}}\left(y_{*}\right)=h_{x, x+\delta_{0}}\left(\lim _{n \rightarrow \infty} y_{n}^{1}\right)=\lim _{n \rightarrow \infty} h_{x, x+\delta_{0}}\left(y_{n}^{1}\right)=h_{x, x+\delta_{0}}\left(y_{1}\right) \neq h_{x, x+\delta_{0}}(0) .
$$

Получаем противоречие.

Покажем теперь, что для пары $(x, y)$, такой, что $F_{L}(x, y)=0$, также верно утверждение леммы. Возьмем произвольную последовательность $\left(y_{n}\right) \rightarrow y, y_{n}<y$ для любого $n$. Так как $F_{L}\left(x, y_{n}\right)<0$, по только что доказанному для любой пары $\left(y_{n}, x\right)$ верно утверждение леммы. Функция $F_{L}(x, y)$ непрерывна по $y$, следовательно, утверждение верно и для пары $(x, y)$.

Лемма 6 доказана.

Лемма 7. Если задача об опасной близости $\left(f, f_{q}, \rho\right)$ сводится к задаче одномерного интервального поиска, то для любой пары $(x, y)$ такой, что $F_{L}(x, y) \leqslant 0$ справедливо равенство

$$
F_{L}(x, y)=F_{L}(1, y)+F_{L}(x, 0)-F_{L}(1,0) .
$$

Доказательство. Для произвольной пары $(x, y) \in[0,1]^{2}$ такой, что $F_{L}(x, y) \leqslant 0$ положим

$$
k=] \frac{1-x}{\delta}\left[, \quad \delta_{0}=\frac{1-x}{k} \leqslant \delta .\right.
$$

Здесь $\delta$ - число из формулировки леммы 5,$] a[-$ наименьшее целое, не меньшее $a$.

Выпишем следующую цепочку равенств, верных в силу леммы 6:

$$
\begin{aligned}
h_{x_{0}, x_{0}+\delta_{0}}(y) & =h_{x_{0}, x_{0}+\delta_{0}}(0), \\
h_{x_{0}+\delta_{0}, x_{0}+2 \delta_{0}}(y) & =h_{x_{0}+\delta_{0}, x_{0}+2 \delta_{0}}(0), \\
& \cdots \\
h_{x_{0}+(k-1) \delta_{0}, x_{0}+k \delta_{0}}(y) & =h_{x_{0}+(k-1) \delta_{0}, x_{0}+k \delta_{0}}(0) .
\end{aligned}
$$

Данная цепочка равенств равносильна цепочке равенств

$$
\begin{aligned}
F_{L}(x, y)-F_{L}(x, 0) & =F_{L}\left(x+\delta_{0}, y\right)-F_{L}\left(x+\delta_{0}, 0\right), \\
F_{L}\left(x+\delta_{0}, y\right)-F_{L}\left(x+\delta_{0}, 0\right) & =F_{L}\left(x+2 \delta_{0}, y\right)-F_{L}\left(x+2 \delta_{0}, 0\right), \\
& \cdots \\
F_{L}\left(x+(k-1) \delta_{0}, y\right)-F_{L}\left(x+(k-1) \delta_{0}, 0\right) & =F_{L}\left(x+k \delta_{0}, y\right)-F_{L}\left(x+k \delta_{0}, 0\right) .
\end{aligned}
$$

По определению $\delta_{0}$,

$$
F_{L}\left(x+k \delta_{0}, y\right)-F_{L}\left(x+k \delta_{0}, 0\right)=F_{L}(1, y)-F_{L}(1,0) .
$$

Таким образом, получаем, что

$$
F_{L}(x, y)-F_{L}(x, 0)=F_{L}(1, y)-F_{L}(1,0),
$$

что и требовалось доказать. 
Перейдем к доказательству необходимости. Пусть

$$
\psi(y)=F_{L}(1, y)-F_{L}(1,0), \quad \psi_{L}(x)=F_{L}(x, 0) .
$$

Тогда по лемме 7

$$
F_{L}(x, y)=\psi(y)+\psi_{L}(x) .
$$

По лемме 3,

$$
F_{R}(x, y)=F_{L}(x, y)+\psi_{R}^{\prime}(x)-\psi_{L}^{\prime}(x) .
$$

Введем обозначение

$$
\psi_{R}(x)=\psi_{R}^{\prime}(x)-\psi_{L}^{\prime}(x)+\psi_{L}(x)
$$

Тогда

$$
F_{R}(x, y)=\psi(y)+\psi_{R}(x)
$$

Необходимость доказана.

\section{Список литературы}

1. Guttman A., R-trees: a dynamic index structure for spatial searching. In: Proc. ACM SIGMOD'84. ACM Press, New York, pp. 47-57.

2. Korn F., Sidiropoulos N., Faloutsos C., Siegel E., Protopapas Z., Fast nearest neighbor search in medical image databases. In: Proc. VLDB'96. Morgan Kaufmann, San Francisco, California, pp. 215226.

3. Roussopoulos N., Kelley S., Vincent F., Nearest neighbor queries. In: Proc. ACM SIGMOD'95. ACM Press, New York, pp. 71-79.

4. Beckmann N., Kriegel H.-P, Schneider R., Seeger B., The R*-tree: an efficient and robust access method for points and rectangles. In: Proc. ACM SIGMOD'90. ACM Press, New York, pp. 322-331.

5. Cheung K. L., Fu A. W., Enhanced nearest neighbour search on the R-tree. ACM SIGMOD Record (1998) 27, №3, 16-21.

6. Benetis R., Jensen C. S., Karciauskas G., Saltenis S., Nearest and reverse nearest neighbor queries for moving objects. The VLDB Journal (2006) 15, №2, 229-249..

7. Song Z., Roussopoulos N., $K$-nearest neighbor search for moving query point. In: Proc. SSTD'01. Springer, London, pp. 79-96.

8. Seidl N., Kriegel H.-P., Optimal multi-step $k$-nearest neighbor search. Proc. ACM SIGMOD'98 154-165.

9. Tao Y., Papadias D., Shen Q., Continuous nearest neighbor search. In: Proc. VLDB'02. Morgan Kaufmann, San Francisco, California, pp. 287-298.

10. Gedik B., Liu L., MobiEyes: A distributed location monitoring service using moving location queries. IEEE TMC (2006) 10, 1384-1402.

11. Atallah M., Dynamic computational geometry. In: Proc. FOCS'83. IEEE Computer Society Press, Los Alamitos, California, pp. 92-99.

12. Bentley J. L., Multidimensional binary search trees in database applications. Commun. ACM (1975) 18, 509-517.

13. Bentley J. L., Friedman J. H., Data structure for range searching. ACM Comput. Surv. (1979) 11, 397-409. 
14. Bolour A., Optimal retrieval algorithms for small regional queries. SIAM J. Comput. (1981) 10, 721-741.

15. Fredman M. L., A lower bound of complexity of orthogonal range queries. J. ACM (1981) 28 , 696-705.

16. Leuker G. S., A data structure for orthogonal range queries. In: Proc. FOCS'78. IEEE Computer Society Press, Los Alamitos, California, pp. 28-34.

17. Leuker G. S., Willard D. E., A data structure for dynamic range queries. Inf. Proc. Lett. (1982) 15, 209-213.

18. Saxe J. B., On the number of range queries in $k$-space. Discrete Appl. Math. (1979) 1, 217-225.

19. Willard D. E., Predicate-oriented database search algorithms, PhD Thesis. Harvard Univ., Cambridge, 1978.

20. Гасанов Э. Э., Кудрявцев В. Б., Теория хранения и поиска информации. Физматлит, Москва, 2002.

21. Лапшов И. С., Динамические базы данных с оптимальной по порядку временной сложностью. Дискретная математика (2008) 20, №3, 89-100.

22. Скиба Е. А., Логарифмическое решение задачи об опасной близости. Интеллектуальные системы (2007) 11, №1-4, 693-719.

23. Снегова Е. А., Случай задачи об опасной близости, сводящийся к одномерному интервальному поиску. Интеллектуальные системы (2009) 13, №1-4, 97-118.

Статья поступила 15.10.2010. 